\title{
Construyendo identidades gais por medio del habla rosa en Santiago de Cali $^{1}$
}

\section{Constructing Gay Identities Through Pink Language in Santiago de Cali}

\author{
Alexánder Ramírez Espinosa \\ Universidad del Valle, Santiago de Cali, Colombia \\ alexander.ramirez.e@correounivalle.edu.co | https://orcid.org/0000-0002-7122-9537
}

\begin{abstract}
Recibido: 19 de junio de 2020. Aprobado: 22 de septiembre de 2020.
DOI: $10.25100 /$ lamanzanadeladiscordia.v15i2.10000

Artículo de investigación
\end{abstract}

¿Cómo citar este artículo? / How to quote this article?

Ramírez, Alexánder. (2020). Construyendo identidades gais por medio del habla rosa en

Santiago de Cali. La Manzana de la Discordia, 15(2),1-29.

10.25100/lamanzanadeladiscordia.v15i2.10000

\footnotetext{
${ }^{1}$ Este artículo es producto de la actualización de datos de un estudio inicial titulado "Modos de creación léxica empleados por un grupo de hombres homosexuales de la ciudad de Cali, en situaciones de habla espontánea", llevada a cabo entre en la Escuela de Ciencias del Lenguaje, Universidad del Valle. Los resultados de dicho estudio se reportaron en Ramírez y Herrera (2018). Las reflexiones del presente artículo corresponden a una actualización de datos del primer estudio y al ejercicio de mediación intracultural que surgió como una necesidad inminente. Se trata de una iniciativa investigativa personal del autor.
} 


\section{Resumen}

El presente artículo de reflexión esboza algunos resultados de un estudio exploratorio y etnográfico sobre las percepciones que los hombres homosexuales de Santiago de Cali, Colombia, tienen sobre su propia variedad lingüística, todo esto a la luz de la literatura sobre creación de identidad y su relación con el lenguaje. Estas reflexiones sugieren que, a pesar de una constante lucha por la reivindicación de sus derechos y el reconocimiento de la sociedad mayoritaria, la comunidad de hombres gais en Santiago de Cali excluye a través de su habla a sus propios pares. Así las cosas, en este artículo se propone un modelo de negociación intracultural que podría constituir una metodología de trabajo para futuros estudios en esta y otras comunidades sexualmente diversas.

Palabras clave: Creación de identidad, Comunidad LGBTQI, Identidades sexuales, Habla gay, Argot gay.

\section{Abstract}

This reflective article outlines some results of an exploratory and ethnographic study on the perceptions that homosexual men in Santiago de Cali have about their own linguistic variety, all this in light of the literature on identity creation and its relationship with language. These reflections suggest that, despite a constant fight for the vindication of their rights and the recognition of the majority society, the community of gay men in Santiago de Cali excludes their own peers through their speech. With this in mind, this article proposes an intracultural negotiation model that could constitute a working methodology for future studies in this and other sexually diverse communities.

Keywords: Identity Creation, LGBTQI Community, Sexual Identities, Gay Speech, Gay slang.

\section{Introducción}

Las reflexiones que presento en este artículo surgieron en el contacto con algunos miembros de la comunidad gay en Santiago de Cali, en el marco de dos proyectos de 
investigación. El primer proyecto fue una investigación sobre la creación léxica en esta comunidad, reportada por Ramírez y Herrera (2018). Tal investigación se enfocó en describir los recursos morfológicos y semánticos utilizados por los hablantes para crear nuevas palabras y nombrar su realidad. Dado que la naturaleza etnográfica de este primer estudio produjo datos interesantes sobre muchos otros aspectos sociales y culturales de la comunidad, se trazó un segundo proyecto de investigación con el objetivo de escudriñar tales datos. En este segundo estudio se buscó que los hablantes se encontraran con un análisis de su discurso para comprender más profundamente la ideología que subyace a sus usos lingüísticos.

En este artículo se redunda en la hipótesis de que los hombres homosexuales en Santiago de Cali experimentan la construcción, tal vez inconsciente, de un conjunto de identidades sexuales no esencializadas, cuyas prácticas culturales y código lingüístico podrían servir como mecanismos de cohesión social. Esta construcción de identidades, sin embargo, está lejos de ser un proceso armonioso. Por el contrario, las diversas entrevistas e historias que subyacen a esta investigación están llenas de expresiones peyorativas contra otros miembros del mismo grupo. Esto sugiere la existencia de tensiones entre varios subgrupos de la comunidad de hombres homosexuales en Santiago de Cali. En otras palabras, aunque todos usan una misma variedad léxica y comparten prácticas sociales similares, existen subgrupos dentro de la comunidad gay, casi a manera de castas, que continúan perpetuando discursivamente relaciones de poder y excluyentes. Sus comportamientos y formas lingüísticas manifiestan la necesidad de una identidad propia, pero en el proceso de construcción de dicha identidad colectiva terminan perpetuando patrones de comportamiento y paradigmas de la sociedad heteronormativa, coartando así las construcciones de identidades individuales de sus miembros.

Con esto en mente, este artículo de reflexión busca ofrecer una perspectiva descriptiva para establecer el panorama del colectivo de hombres gais como un grupo complejo y diverso, cuyos miembros deben ser conscientes y tolerantes de su propia diversidad interna. Se espera que estas reflexiones puedan contribuir al diálogo intracultural entre los miembros de esta comunidad y, por ende, a un posterior diálogo intercultural con la sociedad mayoritariamente heterosexual que los abarca. 


\section{Referentes conceptuales. La construcción de identidades sexuales y los estudios del lenguaje}

La construcción de la identidad es "un proceso subjetivo y emocional que permite a las personas y grupos ubicarse en el mundo" (Guasch, 2000, en Eribon 2000, p. 9). La identidad propicia un sentido de pertenencia, un sentimiento tranquilizador frente a la certeza sobre quiénes somos, de dónde venimos y hacia dónde nos dirigimos. Este proceso subjetivo y personal tiene, sin embargo, un trasfondo colectivo y plural debido a su relación con el lenguaje. Kroskrity (2000) define la identidad como "la construcción lingüística de membresía a uno o más grupos o categorías sociales"² (p.111, traducción personal). Esto refuerza la intrincada relación que existe entre la construcción de identidad y el lenguaje: la identidad es, ante todo, una manifestación lingüística de adhesión a una comunidad, un colectivo o una cultura. Desde esta perspectiva, basta con que un individuo se identifique con una u otra categoría para que se valide su adhesión a la misma, al menos a nivel verbal.

En trabajos anteriores, Kroskrity (1999) ya había descrito la relación entre el lenguaje y las identidades queer/cuir así:

Las identidades de género también se establecen mediante el uso de vocabulario y prácticas discursivas que posicionan al hablante con respecto a los modelos culturales del comportamiento del habla masculino y femenino. Las identidades queer de gays (sic) y lesbianas se producen comunicativamente de una manera similar al redefinir la oposición de género e indexar las diferencias en el habla en un conjunto elaborado de identidades de género subculturalmente reconocidas y de formas apropiadas de manifestarlas. (p.112)

La identidad se crea mediante el uso de ciertas normas lingüísticas, ya que el lenguaje es la herramienta del pensamiento a través de la cual se le da sentido a la realidad y se transforma el mundo. Es una prioridad para los miembros de una comunidad determinada sentirse conectados con el contexto que los rodea, al participar con sus pares en la interacción social. Es, por lo tanto, a través del lenguaje que aquellos que han sido marginados generan empatía, aceptación y sentido de pertenencia para con otros pares. En el caso particular de la comunidad gay, las características lingüísticas en su discurso atestiguan los procesos de construcción de tejido social de individuos cuyo comportamiento

\footnotetext{
2 Todas las traducciones de trabajos previamente no traducidos, son mías.
} 
sociosexual no corresponde al discurso heteronormativo, y cuyos códigos comunicativos son instrumentos de afirmación de su identidad sociosexualmente diversa. Así, el uso de sus propias creaciones permite a los hablantes, en un contexto heteronormativo, desafiar antiguos paradigmas de opresión y discriminación que la sociedad ha impuesto históricamente a los grupos minoritarios o minorizados. Esta misión lingüística libertaria, sin embargo, es un proceso aún en ciernes, ya que el código lingüístico que los define como una comunidad también excluye a sus individuos, categorizándolos en castas superiores e inferiores, y restringe el desarrollo de lo que Kroskrity (1999) llama un conjunto de identidades de género subculturalmente reconocidas.

En este sentido, la apropiación del término queer/cuir, sinónimo en inglés de "extraño", "desviado" o "antinatural", que una vez fue un insulto contra los homosexuales, se produjo a través de un largo proceso de resemantización sociolingüística después de la introducción oficial del término en el mundo académico, gracias a Teresa de Lauretis en 1990. La resemantización del término queer/cuir consiste en la reversión de los matices peyorativos de burla y la adopción de un matiz enfático para definir a todos los individuos que no encajan en las categorías impuestas. A pesar de la tradicional carga homofóbica en el término, la palabra queer ha sido ampliamente aceptada por muchos autores que "insisten en la maleabilidad del término, su capacidad para funcionar como una bolsa en la que se puede encontrar una gran variedad de abusos, y por esta misma razón, una gran variedad de usos rebeldes" (Epps, 2007, p. 223). Así las cosas, el término queer/cuir desafía las ideas preconcebidas de lo que es "normal" y "natural" y lo que no lo es, lo cual no es más que un discurso que obedece a estructuras políticas, religiosas o sociales en las que quien tiene el poder está alineado con lo normal, y el oprimido es el desviado, o el queer. Más particularmente, el término queer/cuir critica las etiquetas socio-sexuales tradicionales, argumentando que las identidades, tan fluidas y diversas como el mismo ser humano, se construyen de manera compleja en la intersección de múltiples discursos.

En términos similares, Weeks (2011) explica que la identidad sexual aparece en la escena "como una forma de afirmar la imagen de sí mismos de aquellos cuyas sexualidades han sido discriminadas por una cultura opresora" (p. 186). La variabilidad de las formas de expresión sexuales está fuertemente ligada a la configuración sociocultural del entorno, 
produciendo así una amplia gama de culturas sexuales (Weeks, 2011; Kinsey et al., 1948; McIntosh, 1968; Gagnon y Simon, 1974; Foucault, 1978). De modo que las sexualidades se configuran de manera diferente en cada cultura, como resultado directo de prácticas sociales complejas que generan un status quo de categorías que algunos dan por sentadas, tales como "lo natural" o "lo normal", categorías que otros buscan desafiar o transgredir. En palabras de Weeks (2011), las sexualidades son el resultado "de definiciones sociales y autodefiniciones, de luchas entre quienes tienen el poder de definir y regular y quienes se resisten" p. 193).

En este sentido, la creación de una identidad, y la manifestación verbal de pertenencia a ella, constituyen la piedra angular para el nacimiento de una nueva comunidad con una cultura propia, una de camaradería y resistencia. Vale la pena señalar que la identidad homosexual, o cualquier otra identidad diversa, "se establece firmemente cuando un individuo ha desarrollado una autoimagen gay positiva" (Waldner-Haugrud y Magruder, 1996, p.314). Sin embargo, las jergas específicas de la comunidad homosexual son "un repertorio de recursos semióticos culturalmente formados para indexar un rango de identificaciones y producir de manera performativa una Identidad no esencializada" (Gray, 2016, p. 228). Dichas jergas pueden fomentar el uso de normas lingüísticas que atacan la diversidad inconscientemente para cohesionar una identidad gay. Esto es lo que Castelar (2015) llama "discurso de odio", un conjunto de actos lingüísticos y de performatividad que "operan como un dispositivo de modificación corporal, que somete a aquellos seres considerados inferiores y los disciplina. La sujeción va por la vía de la agresión verbal directa, que poco a poco va conformando seres inseguros, temerosos, culpables" (p.71).

\section{El Habla rosa}

El Habla rosa (Ramírez y Herrera, 2018) es el conjunto de formas y expresiones lingüísticas utilizadas en el habla espontánea por hombres homosexuales, aunque en la literatura académica también existen términos como Habla Lavanda, o Gayspeak. Estas hablas se caracterizan por ser disruptivas (Harvey, 2000), exageradas (Hayes, 2006) y transgresoras de las reglas gramaticales, así como de las reglas sociales (Herrera y Ramírez, 2018). El término Habla rosa nace de la necesidad de bautizar a un sociolecto particular en 
Santiago de Cali que, como señala Gray (2016), es "cultural, histórica, geográficamente específico" (p.228) como las jergas de otras comunidades homosexuales.

Autores como McIntosh (1968) y Cameron y Kulik (2003) señalan que el interés por el habla homosexual se remonta al siglo XVIII en fuentes que sugieren que "los homosexuales utilizan el lenguaje de maneras muy particulares" (Gray, 2016, p. 227), y que el discurso en hombres gais y travestis enmarca una amplia gama de variaciones argóticas incluso más evidentes "que con lesbianas y otras minorías sexuales" (p. 227). De hecho, el secretismo, la confidencialidad, la búsqueda de identificación y cohesión comunitaria, entre muchas otras características de la comunidad gay, hacen de su discurso una fuente rica de creación léxica e identitaria (Ramírez y Herrera, 2018).

\section{La necesidad de una mediación intracultural}

Sin embargo, ¿cómo podría un individuo desarrollar una autoimagen positiva mientras es denigrado por la comunidad que la rodea? Este tipo de hostilidad en la comunidad de hombres homosexuales en Santiago de Cali puede contrarrestarse mediante la comunicación y la mediación intracultural.

Kecskes (2018) define la comunicación intracultural como aquella que "ocurre en interacciones entre miembros de una comunidad de habla que comparte una misma lengua materna relativamente definible, y que sigue unas convenciones lingüísticas y de uso con opciones y preferencias individuales" (p. 6). En este caso, los hombres homosexuales de Cali pertenecen, principalmente, a la cultura colombiana y caleña, y comparten la variedad del español colombiano como su lengua materna. De acuerdo con Kecskes (2018), la comunicación intracultural tiene que ver con "el grado de confianza en el terreno común" (p. 15). Woolard y Schieffelin (1994, en Kecskes, 2018) definen la ideología del lenguaje como:

las redes de creencias que poseen los usuarios de una lengua, ya sea de manera tácita o explícita, sobre la lengua misma y su relación con otros aspectos de sus entornos, especialmente otros individuos y grupos sociales, que se derivan bien sea de la enseñanza explícita o de la socialización implícita. Debido a las conexiones existentes entre la lengua y los usuarios o grupos sociales, las ideologías del 
lenguaje están íntimamente conectadas a las macroestructuras de poder y privilegio. (p. 63)

\section{Metodología}

Este artículo retoma los resultados de un estudio etnográfico (Ramírez y Herrera, 2018), descriptivo e interpretativo llevado a cabo en tres etapas, cuyo objetivo fue obtener una caracterización léxica del habla espontánea utilizada por hombres homosexuales en Santiago de $\mathrm{Cali}^{3}$. Dicho estudio corresponde a una mirada del habla espontánea en discusiones callejeras, discotecas, sitios virtuales anónimos y lugares de encuentro, a menudo clandestinos, en donde los miembros de la comunidad gay desarrollan sus prácticas culturales. Los resultados de la primera investigación sentaron las bases para establecer las estrategias utilizadas por los hablantes para nominar su entorno y su realidad. Además, este primer estudio me llevó a preguntar qué es lo que realmente nos dicen estas palabras sobre las personas que las usan y qué revela este modo de hablar en particular sobre las normas de comportamiento dentro de la comunidad de habla.

Con esto en mente, se emprendió una nueva investigación, con el objetivo de analizar la construcción de la identidad sexual a través del uso de un código lingüístico particular. Este segundo estudio consistió en llevar los resultados de la primera investigación a los miembros de la comunidad y documentar sus reacciones y concepciones frente a la disección de su propio discurso. La siguiente sección de este artículo esboza los resultados principales de este segundo estudio.

\footnotetext{
${ }^{3}$ Para la etapa inicial, se utilizaron métodos clásicos de recolección de corpus, como la observación directa del habla espontánea, entrevistas semiestructuradas y preguntas sobre ítems léxicos concretos, así como las técnicas de observación participante propuestas por DeWalt y DeWalt (2002). Se tuvieron en cuenta las interacciones en medios electrónicos, tales como redes sociales y las salas de chat, que son por naturaleza medios de habla espontánea que "ponen en circulación nuevos conceptos y vocabularios promoviendo un mayor uso de los mismos" (Gray, 2016, pág. 227). En la recolección del corpus participaron 60 hombres homosexuales con edades entre 18 y 45 años. Inicialmente, se recogieron doscientos ochenta y cuatro (284) términos de 25 horas de grabaciones de audio y, en promedio, 10 horas de interacciones en chats y redes sociales. La segunda etapa estuvo dedicada a depurar el corpus de términos idiolectales no representativos. Para este propósito, se pidió a diez nuevos informantes que confirmaran el uso de los términos. El corpus final, compuesto por doscientas seis (206) entradas, fue construido con los términos reconocidos por al menos 7 de 10 informantes. Un primer análisis permitió organizar los términos en seis categorías: 1. Insultos; 2. Denominación y Auto-denominación; 3. Sexo y sexualidad; 4. Lugares de encuentro; 5. Actividades de la comunidad y 6. Locuciones. Finalmente, la tercera etapa del estudio consistió en analizar y describir los mecanismos semánticos y morfológicos de la creación léxica, según la propuesta de Montes (1983).
} 
Para este nuevo estudio se seleccionaron 50 individuos, de los cuales 32 ya habían participado como informantes en la investigación antecedente. Estos participantes fueron los encargados de invitar a sus amigos y conocidos a hacer parte del estudio, y fue así como se completó el grupo de 50 individuos. Para la selección de los participantes, se establecieron los criterios de procedencia y residencia en la ciudad de Santiago de Cali. Además, todos los participantes debían autoidentificarse como hombres homosexuales. En el grupo de hombres entrevistados hubo siete individuos que se autoidentificaron como afrodescendientes, aunque la adscripción étnica-racial no fue un criterio de selección; los 43 participantes restantes son mestizos. Finalmente, y aunque la clase social tampoco fue un criterio de selección, todos los participantes del estudio se ubican en diferentes estratos socioeconómicos entre los estratos 3 y 5 , por lo cual se puede hablar de una muestra poblacional de clase media y media-alta.

A través de cinco grupos focales, se presentó el análisis y resultados de la investigación del Habla rosa en Santiago de Cali, de manera que los participantes estuvieran frente a una disección de su habla y así explorar su motivación semántica. Las discusiones resultantes de los grupos focales se transcribieron para facilitar la codificación de categorías emergentes; finalmente, los datos resultantes se triangularon para llegar a una síntesis de las reflexiones por parte de los hablantes.

\section{Una mirada al espejo: reacciones de los hablantes frente a su discurso}

Primero, se les preguntó a los participantes sobre el origen de las formas de autodenominarse y denominar a otros miembros de su la comunidad, usando los términos bajo la categoría Denominación y Autodenominación descrita por Ramírez y Herrera (2017). Todos los participantes manifestaron usar la palabra gay como un amplio hiperónimo bajo el cual se clasifican a sí mismos y su sexualidad diferenciada. Sin embargo, un pequeño número de los participantes (seis en total) afirma que se sienten incómodos con la palabra gay, ya que generalmente se asocia con un estilo de vida sórdido e indecoroso; así lo expresó uno de los participantes, a quien he denominado Marcos ${ }^{4}$ : "Soy homosexual porque me atrae otro hombre, pero no me considero gay porque busco estar al

\footnotetext{
${ }^{4}$ Los nombres de los participantes se han cambiado para proteger su intimidad. 
margen de lo que implica la cultura gay y sus prácticas" (grupo focal, 10 de noviembre de 2018). Las prácticas a las que se refiere Marcos y cinco participantes más del estudio aparecieron sutilmente a lo largo de los grupos focales: la asistencia a discotecas gais, la visita a sitios de encuentros sexuales como saunas o salas de internet con cabinas privadas, el uso de manillas con la distintiva bandera arcoíris, o el cruising, solo por mencionar algunas. No obstante, es importante aclarar que estas no son prácticas necesariamente exclusivas de los homosexuales. Estas prácticas incluyen también la forma de hablar, en lo que Kroskrity (1999) llama un "trabajo comunicativo estratégico que les permite esgrimir o suprimir identidades específicas en la interacción" (pág. 112), con el fin de ser —o no serclasificado y evaluado por otros interactuantes.

Luego, se presentaron las categorías Sexo y Sexualidad, Insultos y Locuciones a los participantes de los grupos focales. Una de las primeras impresiones en los cinco grupos focales tuvo que ver con la sorpresa de los hablantes ante la cantidad de términos despectivos presentes en su propio discurso. Aparte de la categoría de Insultos, las categorías de Denominación y Autodenominación, Sexo y Sexualidad, así como la de Locuciones cuentan con abundantes imágenes estereotipadas y voces peyorativas que se usan en contra de otros miembros sexualmente diversos de la comunidad LGTBQI, e incluso contra otros hombres homosexuales.

El análisis de estos términos despectivos sugirió una marcada tendencia hacia la binariedad de lo masculino y lo femenino. En el corpus hay insultos, por ejemplo, para hombres homosexuales con comportamientos femeninos, así como para hombres homosexuales con comportamientos masculinos. Esto revela dos subgrupos de personas dentro de la comunidad: por un lado, un grupo de hombres homosexuales que desprecian las conductas y características femeninas en sus pares; por otro lado, hay un grupo de personas que consideran que ser gay va de la mano con la feminidad, y por lo tanto, cualquier comportamiento masculino no es más que una fachada de un hombre gay "enclosetado" que merece ser insultado y expuesto. También existen términos ofensivos contra las personas que se definen como bisexuales, ya que son considerados como individuos que no han aceptado su homosexualidad por completo, o que utilizan tal 
autodenominación como un eufemismo lingüístico menos desdeñado dentro de la comunidad heteronormativa.

Se preguntó a los participantes sobre la motivación detrás del uso de estos insultos y sus respuestas permitieron establecer una discusión franca sobre diversas temáticas controversiales como el género, la preferencia sexual y la construcción de masculinidades y feminidades. Esta discusión se redujo a tres posturas principales: primero, algunos hombres homosexuales que agencian en sí mismos una conducta masculina justifican su uso de términos ofensivos hacia hombres homosexuales femeninos, diciendo que estos últimos desdibujan la figura masculina. Para estos hombres gais que se llaman así mismos "serios", la preferencia sexual hacia otros hombres no implica la adopción de conductas femeninas. Para estos hombres, ser serio implica ostentar comportamientos culturalmente asociados a lo masculino, como tener movimientos fuertes y bruscos, tener voz gruesa, evitar el uso de simbología y parafernalia asociados a la homosexualidad (como la bandera arcoíris o el color rosado). Así lo manifestó, por ejemplo, unos de los informantes a quien he llamado Adriano: "Hay manes gay (sic) que se dicen serios en el chat o en Tinder y cuando uno los llama...¡Noooo! ¡Son más mujer que mi mamá! Si uno es serio no tiene por que notársele la maricada, ni andar apretadito en la calle, ni hablar delgadito" (grupo focal, 10 de noviembre de 2018). Nótese que las palabras de este informante sugieren que ser serio implica no ser percibido como homosexual. Al igual que Adriano, seis informantes más mencionaron estrategias que se usan como ejercicios de la "seriedad", entre los cuales se destacan mostrar interés por el fútbol, no rodearse de otros homosexuales considerados "evidentes", vestir ropa holgada, no frecuentar sitios gais y cuidarse conscientemente las expresiones del habla asociadas a los homosexuales (como la efeminización, el uso de vocablos argóticos y la gesticulación exagerada).

Aquellos que se autodefinen como gais serios ven el uso de manerismos como una transgresión forzada e irreal de la figura idealizada del macho. Usan palabras peyorativas para referirse a otros hombres homosexuales que tienen voz aguda, que usan cierto tipo de ropa o colores considerados femeninos, que se mueven, caminan o gesticulan de una manera que la cultura heteronormativa dominante ha considerado exclusiva para las mujeres y desprecian estos comportamientos por considerarlos una pantomima forzada que 
transgrede el status quo de lo masculino y lo femenino. En otras palabras, este primer grupo de hombres gais cree que todos los hombres homosexuales son masculinos, y que sus características femeninas son conductas postizas y exageradas, y que deberían "portarse machitos" por respeto a los demás. Esto, por supuesto, es una postura sesgada que pierde de vista la complejidad del tema.

En contraste, algunos hombres homosexuales que ostentan comportamientos más delicados consideran que la feminidad es inherente a ser homosexual. Para algunos de ellos (cinco en total), el hecho de que un hombre gay se vea masculino surge del deseo de camuflar su sexualidad. Por lo tanto, desdeñan a los homosexuales que trabajan en busca de un cuerpo masculino fuerte, o que se involucran en actividades o deportes que han sido clasificados culturalmente como rudos. Este grupo considera que estos homosexuales con comportamientos masculinos son "gais enclosetados" que adoptan falsas conductas por miedo a ser descubiertos en su preferencia sexual. Por ejemplo, un participante del estudio, a quien he llamado Efraín, dice: "uno ve mucho gay camuflado sobre todo en los gym, eso sí, póngale la firma que allá abundan las 'muscu-locas' tratando de sacar cuerpo pa' verse machitas y que no se les note el cuento" (grupo focal, 25 de enero de 2019). Sin embargo, todo esto es contradictorio con el hecho de que el comportamiento masculino es, a su vez, una característica muy atractiva y altamente deseada dentro de la comunidad gay cuando se trata de establecer relaciones sociales, románticas o sexuales, según lo revelan muchas otras expresiones en el corpus lingüístico, así como investigaciones anteriores (Castelar, 2015).

Los demás participantes defendieron un tercer punto de vista más conciliatorio, desde el cual argumentan que las categorías de lo masculino y lo femenino no son estrictamente excluyentes en las construcciones de género. Desde esta visión, cada individuo representa una construcción particular y subjetiva de ser y de comportarse, una identidad sexual que no puede ser camuflada y que será percibida culturalmente como más inclinada hacia lo masculino o lo femenino. En otras palabras, los que esgrimen este argumento entienden la construcción de la identidad sexo-genérica como un continuum fluido, un concepto fundamental en la teoría Queer, a lo largo del cual cada individuo encuentra un lugar, ya sea más lejos o más cerca de los extremos masculino o femenino. Un participante, a quien llamaré Jerónimo, nos explica su visión así: “Uno es lo que es... yo, 
por ejemplo, les puedo parecer muy maniquebrado a algunos y muy serio a otros, pero yo no siento que pueda ni portarme más machito ni tampoco mariquiarme más, o sea, yo soy como soy, y cualquier intento por amanerarme o ponerme más serio va a terminar siendo un teatro mal hecho" (grupo focal, 09 de febrero de 2019). Desde este punto de vista, y según los participantes en los grupos focales, el homosexual de conductas masculinas no está escondiendo su preferencia sexual, ni el homosexual de comportamientos femeninos está parodiando a las mujeres, sino que ambos casos son construcciones individuales respetables en dos puntos opuestos del continuum, en medio de los cuales hay tantos matices como personas. La imagen 1 ilustra este punto de vista:

Imagen 1. Continuum de masculinidad y feminidad

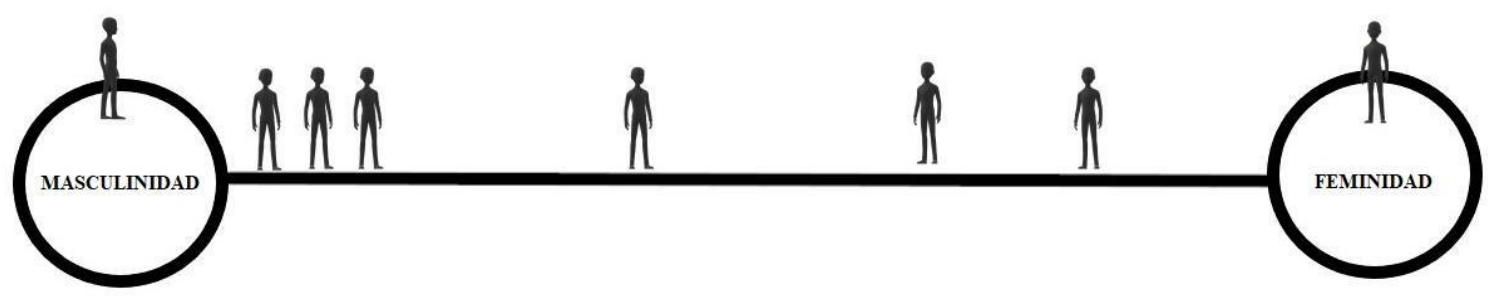

Fuente: Propuesta del autor.

La idea que subyace a este gráfico se basa en los principios de la teoría Queer, que rechaza la clasificación de individuos en categorías universales y fijas. Estas categorías fijas, a menudo binarias, restringen el comportamiento humano a las imposiciones culturales que hacen que la heterosexualidad y la heteronormatividad sean obligatorias o, en este caso, que la masculinidad y la feminidad sean excluyentes la una de la otra. En el caso de muchos hombres homosexuales en Santiago de Cali, su discurso podría estar reproduciendo la idea de un binarismo absoluto que opone a los gais 'machos' contra los gais 'maricas'; tal binarismo perpetúa visiones estrechas y restrictivas como las de hombremujer o heterosexual-homosexual, olvidando toda una serie de posibles comportamientos e identidades intermedias; Guasch (2000, en Eribon, 2000) lo plantea así:

$\mathrm{Y}$ es que se tolera a homosexuales y lesbianas siempre y cuando sean discretos. Probablemente la peor consecuencia sea que algunos homosexuales y algunas lesbianas están dispuestos a reproducir el modelo normativo con el fin de obtener 
respeto social. El precio implica esconder, entre otros, a los chaperos, a las locas y a las camioneras [...] De este modo triunfa una sofisticada tecnología de control social que pretende difundir el modelo heteronormativo entre los y las disidentes. (p10)

A los participantes también se les mostró que su léxico está cargado de términos despectivos contra otros grupos sexualmente diversos:

- Lesbianas: los términos utilizados para llamar a las mujeres lesbianas suelen ser alusivos a comportamientos o profesiones masculinos, como por ejemplo las palabras 'machorra' o 'caminonera. También se destaca el uso de metáforas animalísticas despectivas como 'gallinas', 'gatas' o 'suricatas', que se usan indistintamente contra mujeres lesbianas o heterosexuales, ubicándolas en una categoría antagónica.

- Transgénero y travestis: la burla hacia estos miembros de la comunidad es más común cuando no se ajustan a los estándares de belleza que coinciden con la imagen de mujer voluptuosa impuesta por los medios; así, se usa la expresión 'careca', que lingüísticamente corresponde a una paranomasia ${ }^{5}$ de la expresión 'cara de caballo', para referirse a una mujer trans cuyas facciones no son delicadas. De manera similar, se hace referencia a los travestis como "versiones baratas" de una persona transgénero, y se les denomina con la paranomasia 'trabestia' que combina las palabras 'travesti' y 'bestia'. Esto demuestra la ignorancia de la terminología LGBTIQ por parte de muchos miembros de la misma comunidad. Además, varias palabras en el léxico gay de Cali, como 'putitranny', se usan para asociar erróneamente a las mujeres transgénero y travestis con el trabajo sexual, aunque se ha comprobado que este no es el caso de todas (Urrea y La Furcia, 2014).

- Homosexuales afrodescendientes o indígenas: otras palabras y expresiones de desprecio dentro del corpus lingüístico en Santiago de Cali se usan contra homosexuales provenientes de grupos étnicos minoritarios, como es el caso de 'pelimalditas' para referirse a los homosexuales afrodescendientes, o 'aldeanas' para homosexuales indígenas. Los participantes en los grupos focales creen que este tipo de discriminación ocurre por una

\footnotetext{
${ }^{5}$ La paranomasia es una figura del lenguaje en la que se usa la estructura morfológica o fonológica de una o más palabras para producir efectos lúdicos y estilísticos (Ramírez y Herrera, 2018).
} 
concepción errónea de que ser una minoría sexual ya es un estigma, por lo tanto, ser de una minoría sexual y étnica a la vez es, como lo llamaron ellos, una 'maldición gitana'.

- Adultos mayores homosexuales: mientras que la belleza y la juventud son muy valoradas dentro de la comunidad gay, los adultos mayores homosexuales son despreciados a través del uso de palabras burlonas e insultos. La jerga de los hombres homosexuales en Santiago de Cali sugiere que la cultura gay está ligada a la juventud, y que los homosexuales que han alcanzado la tercera edad deben evitar la promulgación de su identidad sexual. Uno de los participantes del estudio, a quien llamaré Franco, lo manifiesta así: "Mirá, no hay nada más feo que una marica arrugada y destruida, o sea, si ya estás 'vejeta' tenés que dejar de mariquiarte, ya no estás pa' andar en las discotecas ni pa' ponerte shortcitos calientes" (grupo focal, 09 de febrero de 2019).

Finalmente, muchas palabras y expresiones encontradas en el corpus evidencian una marcada exclusión, al menos a nivel discursivo, contra los hombres homosexuales de escasos recursos, contra aquellos que prefieren el rol sexualmente pasivo y contra todos aquellos que no cumplen con los estándares de belleza que la comunidad ha impuesto en términos de tamaño y peso, de las marcas de ropa que usan, los sitios que frecuentan, o incluso la música que escuchan. No obstante, la imagen idealizada que sugiere el habla rosa en Santiago de Cali, aquella del hombre homosexual joven, de piel clara, de cuerpo atlético, con poder adquisitivo evidente en sus atuendos, que escuchan música electrónica y pop anglo porque hablan una o más lenguas extranjeras, no es el común denominador en los grupos de participantes estudiados en la primera ni en la segunda investigación. Se trata, a mi parecer, de una imagen comercial que no los representa pero que se verbaliza como una meta o un requisito de membresía en la comunidad.

Es evidente que, a pesar del deseo explícito de fortalecer su comunidad, este grupo estudiado de homosexuales en Santiago de Cali se definen y diferencian constantemente en contraposición a las mujeres, los viejos, los pobres, los indígenas y los afrodescendientes. Esto es lo que Guasch (2000, en Eribon 2000) llama una identidad gay "pobre en matices, conservadora y excluyente, pero que vende bien. Se trata de vender mitos que la gente está dispuesta a comprar: el mito de la juventud, el de la belleza, el de la distinción. El mercado gay (y en especial la prensa gay) se centran en un único modelo homosexual, aunque, en La Manzana de la Discordia • Vol. 15. No. 2 • junio-diciembre 2020 • pp. 64-86 • E-ISSN 2500-6738 
realidad, existen centenares de modelos" (p.10). Es un tipo de identidad artificial y estereotipada que, irónicamente, no da lugar a la diversidad, como si fuera menos gay quien no se ve exactamente como el estereotipo contra el que luchan.

Después de encarar este análisis, los participantes llegaron a dos conclusiones principalmente: primero, que no eran muy conscientes de la variante léxica que habían estado creando a lo largo del tiempo, lo cual es típico en los estudios lingüísticos; y segundo, que a través de su forma de hablar, siguen reproduciendo patrones de comportamiento binarios que contradicen su búsqueda de respeto hacia la diversidad. En otras palabras, abogan políticamente por la homosexualidad como una forma válida de diversidad sexual, pero desde el punto de vista lingüístico desdeñan la diversidad dentro de su propia comunidad.

Un común denominador en los grupos focales fue la expresión de preocupación por parte de los participantes frente a no querer ser vistos como un colectivo discriminador, incluso si su habla denota matices excluyentes contra unos otros que son más cercanos que lejanos. Al respecto, Butler (1997) afirma que el insulto es "una de las condiciones a través de las cuales un sujeto se constituye en el lenguaje"; en otras palabras, el insulto y las voces peyorativas en este grupo estudiado cumplen una función de interpelar y crear discursivamente al otro "bajo el riesgo de inaugurar a un sujeto en el discurso que viene a usar el lenguaje para contrarrestar la ofensa" (Butler, 1997, p. 2). Si bien se interpela al otro desde la ofensa, los participantes han manifestado no querer ser excluyentes en la realidad.

Estas características están también presentes también en sociedades que suponíamos más avanzadas en temas de diversidad sexual; Guasch (2000, en Eribon 2000) explica que dentro del universo gay francés hay pensamientos que se oponen a la pluralidad y diversidad de las identidades. Para Guasch, estos pensamientos imponen íconos de consumo de una única forma de identidad gay que se convierten en referentes simplistas, estandarizados e irreflexivos para la mayoría heteronormativa. En otras palabras, los homosexuales podrían estar alimentando los estereotipos que otros se han formado sobre ellos, y en el caso particular de Santiago de Cali, lo están haciendo a través de su léxico. 
La libre expresión de las identidades sexuales se convierte en un acto problemático en entornos culturales donde la represión ha sido el status quo; y es aún más problemático cuando la misma comunidad de individuos marginados ha establecido distinciones jerárquicas, con palabras peyorativas para llamar a los que consideran inferiores, promoviendo una distribución desigual de poderes, en la que los privilegiados tienen la posibilidad de categorizarse a ellos mismos y a otros (Foucault, 1966; Castelar, 2015).

Podría interpretarse que estas formas linguiísticas excluyentes son un síntoma de miedo; Weeks (2011) menciona que las identidades sexuales, y yo agregaría su manifestación lingüística, son "siempre e inevitablemente relacionales: existen debido a la presencia de un otro a menudo temido o amenazante" (p. 188). En otras palabras, la agresividad en el Habla rosa de Santiago de Cali podría ser un síntoma de la necesidad de reconocimiento que, desafortunadamente, estereotipa a otros subgrupos que tienen la misma necesidad.

Con esto en mente, se invitó a los participantes a identificar y clasificar, en un papelógrafo, algunas de las ideas polarizadas y conflictivas que subyacen a su léxico. Asumiendo un rol de mediador, mi labor fue ayudarles a sintetizar sus ideas en una serie de frases que se colocaron en dos extremos opuestos. El siguiente paso fue proporcionar una idea conciliatoria en medio de ambos extremos, basada en el reconocimiento de las otras posturas y de referentes teóricos, a fin de crear ese terreno común básico que propone Kecskes (2018). La siguiente tabla presenta una muestra de este ejercicio:

Tabla 1. Ejercicio de mediación intracultural

\begin{tabular}{|l|l|l|}
\hline \multicolumn{1}{|c|}{ Extremo A } & \multicolumn{1}{|c|}{ Idea conciliatoria } & \multicolumn{1}{c|}{ Extremo B } \\
\hline $\begin{array}{l}\text { El comportamiento } \\
\text { femenino es inherente a } \\
\text { todos los hombres } \\
\text { homosexuales. Cualquier } \\
\text { rasgo de la masculinidad } \\
\text { es un intento de camuflar } \\
\text { su verdadera identidad. }\end{array}$ & $\begin{array}{l}\text { La construcción de las masculinidades y } \\
\text { las feminidades son independientes de la } \\
\text { atracción sexual. Dictaminar cómo deben } \\
\text { comportarse los hombres responde a una } \\
\text { percepción heterocéntrica en la que los } \\
\text { comportamientos no convencionales } \\
\text { pueden ser despreciados y rechazados sin } \\
\text { ninguna consecuencia (López, 2008). }\end{array}$ & $\begin{array}{l}\text { Todos los hombres } \\
\text { homosexuales tienen que } \\
\text { comportarse de una } \\
\text { manera masculina, } \\
\text { porque los rasgos } \\
\text { femeninos son una } \\
\text { transgresión postiza que, } \\
\text { desdibuja la imagen del } \\
\text { hombre. }\end{array}$ \\
\hline
\end{tabular}




\begin{tabular}{|c|c|c|}
\hline Extremo A & Idea conciliatoria & Extremo B \\
\hline $\begin{array}{l}\text { Los homosexuales tienen } \\
\text { que ser políticamente } \\
\text { activos y participar en } \\
\text { desfiles, marchas y } \\
\text { manifestaciones. La } \\
\text { omisión de este deber es } \\
\text { un intento de ocultar su } \\
\text { preferencia sexual. }\end{array}$ & $\begin{array}{l}\text { Si bien es normal buscar la sindicalización } \\
\text { con fines políticos, en aras del } \\
\text { reconocimiento y de la lucha legítima por } \\
\text { los derechos, cada persona expresa su } \\
\text { identidad de diferentes maneras. La } \\
\text { identidad política es solo una entre muchas } \\
\text { otras identidades que un individuo adopta } \\
\text { o reprime de manera estratégica e } \\
\text { individual (López, 2008). }\end{array}$ & $\begin{array}{l}\text { Los homosexuales } \\
\text { políticamente activos } \\
\text { alimentan el estereotipo } \\
\text { gay, pues son personas } \\
\text { con un comportamiento } \\
\text { obsceno, escandaloso y } \\
\text { controvertido, que } \\
\text { fomenta la imagen } \\
\text { negativa que la sociedad } \\
\text { tiene sobre nosotros. }\end{array}$ \\
\hline $\begin{array}{l}\text { Los manierismos son un } \\
\text { rasgo para los jóvenes } \\
\text { gais. Los adultos mayores } \\
\text { gais se ven ridículos } \\
\text { siendo femeninos, y } \\
\text { deberían comportarse } \\
\text { "serios". }\end{array}$ & $\begin{array}{l}\text { Si bien la edad es una categoría que } \\
\text { implica cambios en el comportamiento, la } \\
\text { idea de distribuir la masculinidad o la } \\
\text { feminidad exclusivas en grupos etarios es } \\
\text { absurda. Las identidades son fluidas y no } \\
\text { tenemos solo una, sino múltiples niveles de } \\
\text { identidades cambiantes e incluso } \\
\text { conflictivas, que surgen en contextos } \\
\text { diferentes (Wardhaugh y Fuller, 2014). }\end{array}$ & $\begin{array}{l}\text { Los adultos mayores gais } \\
\text { que ya han alcanzado } \\
\text { una estabilidad } \\
\text { económica tienen el } \\
\text { derecho de actuar como } \\
\text { les plazca. Los jóvenes } \\
\text { amanerados avergüenzan } \\
\text { al resto de la comunidad. }\end{array}$ \\
\hline $\begin{array}{l}\text { Todos los homosexuales } \\
\text { deben frecuentar las } \\
\text { discotecas gais, bares y } \\
\text { saunas porque son los } \\
\text { únicos lugares de reunión } \\
\text { para la comunidad. }\end{array}$ & $\begin{array}{l}\text { Los lugares de reunión proporcionan un } \\
\text { entorno para que las comunidades } \\
\text { desarrollen prácticas culturales y } \\
\text { fortalezcan el sentido de pertenencia. Sin } \\
\text { embargo, una comunidad debe evitar el } \\
\text { "neo-tribalismo" (Weeks, 2011) y } \\
\text { trascender la idea de que la comunidad } \\
\text { solo se construye en instalaciones físicas. } \\
\text { El objetivo es establecer una comunidad } \\
\text { crítica, en términos de Foucault (1966), } \\
\text { que permita todo tipo de subjetividades, } \\
\text { incluida la participación o la evitación de } \\
\text { prácticas culturales preestablecidas. }\end{array}$ & $\begin{array}{l}\text { Los sitios de encuentro } \\
\text { gay son lugares sórdidos } \\
\text { en los que se promueven } \\
\text { diversos tipos de } \\
\text { comportamientos } \\
\text { clandestinos. Por lo } \\
\text { tanto, un homosexual } \\
\text { "serio" solo debe ir a } \\
\text { sitios "directos". }\end{array}$ \\
\hline $\begin{array}{l}\text { El bisexualismo es un } \\
\text { eufemismo que algunos } \\
\text { homosexuales usan porque } \\
\text { tienen miedo o se } \\
\text { avergüenzan de asumirse } \\
\text { abiertamente como } \\
\text { homosexuales. }\end{array}$ & $\begin{array}{l}\text { El bisexualismo es solo otra forma de } \\
\text { autoidentificación, utilizada por aquellos } \\
\text { que se sienten atraídos sexualmente por } \\
\text { hombres y mujeres, independientemente de } \\
\text { la cuantificación de tal atracción. En ese } \\
\text { sentido, atacar a quienes reclaman una } \\
\text { identidad bisexual es equivalente a } \\
\text { imponer una categoría basada en la } \\
\text { realidad personal como la única opción } \\
\text { válida. }\end{array}$ & $\begin{array}{l}\text { Los hombres bisexuales } \\
\text { son personas vacilantes, } \\
\text { confundidas, que no son } \\
\text { lo suficientemente } \\
\text { valientes para tomar una } \\
\text { decisión sobre lo que } \\
\text { realmente quieren. }\end{array}$ \\
\hline
\end{tabular}

Fuente: Grupos focales, noviembre de 2018 a febrero de 2019. 
Luego de este ejercicio y de las diversas discusiones planteadas en los grupos focales, muchos de los participantes expresaron satisfacción y asombro al escuchar otras posturas sobre temas que daban por sentados de manera viciada. Todos los participantes reconocieron que su Habla rosa, tan jocosa y necesaria en la cohesión de su comunidad, ostenta elementos que estereotipan la raza y la clase, forjando una serie de concepciones erróneas. En este sentido, Harsin (1991) llama la atención sobre el hecho de que "la mayoría de jóvenes homosexuales no se crían en un entorno en el que el desarrollo de su homosexualidad sea reconocido, y mucho menos alentado. No es inusual que los hombres se hayan identificado recientemente como gay (sic) no tengan idea de lo que significa ser gay" (p. 31). En otras palabras, los jóvenes que "salen del closet", aterrizan en alguno de los subgrupos de la comunidad gay y heredan su jerga y sus ideologías, sin una comprensión informada de que su comportamiento y sus prácticas culturales reproducen los mismos paradigmas de rechazo que ellos mismos han sufrido.

\section{A manera de conclusión}

Los resultados de este estudio mostraron, por un lado, que los todos los participantes usan o reconocen los términos y expresiones del Habla rosa de Santiago de Cali, lo cual sugiere la construcción colectiva de un mecanismo cohesionador a través del habla. Por otro lado, sin embargo, todos los participantes afirmaron que no eran conscientes de la carga peyorativa presente en la mayoría de términos. Esta manera de definirse en contraposición verbal —a veces agresiva - a los otros, ha producido una gran cantidad de vocablos que establecen relaciones de poder desde lo discursivo. Los participantes manifestaron su sorpresa y preocupación frente a los matices excluyentes de su jerga, pues todos expresaron constantemente su deseo por construir una comunidad incluyente, respetuosa y diversa.

En este sentido, el ejercicio de mediación intracultural resultó ser muy útil para incentivar la conciencia sobre la otredad y las formas lingüísticas que se usan para nominarla, así como las formas de autonominarse con respecto a los otros. El análisis consciente y colectivo de las formas de hablar permitió a los participantes identificar la reproducción de patrones binarios que se heredan de la sociedad mayoritariamente heterocisnormada y que podrían socavar la construcción de un colectivo sólido sobre las bases del respeto. Asimismo, el diálogo intracultural que se promovió durante y después de los 
grupos focales permitió a los participantes negociar percepciones, manifestar argumentos e incluso reconsiderar prejuicios.

Quedan aún cabos sueltos y mucho trabajo por hacer con respecto al discurso de las comunidades minoritarias sexualmente diversas. Este trabajo es un esbozo inicial de la realidad lingüística de cohesión y resistencia dentro de un grupo de hombres homosexuales en Santiago de Cali; pero falta completar un panorama más amplio del vasto conjunto de realidades lingüísticas y sexualmente diversas en Santiago de Cali: las de las lesbianas, las mujeres y hombres transgénero, los bisexuales e intersexuales, etc., y verificar si la creación léxica dentro de estos subgrupos también fomenta discursos excluyentes. Una mirada completa al panorama lingüístico de esta comunidad permitiría seguir estudiando la relación entre discurso y creación de identidades individuales y colectivas. Asimismo, las descripciones del léxico de diversos colectivos permitirían la mediación intracultural que se propone en estos acápites y sería la base sobre la cual se podría establecer un diálogo intercultural con la comunidad predominantemente heterosexual. Esto implica la adquisición de una literacidad sexual, que tiene que ver con "aprender a disfrutar y apreciar las identidades sexuales [...]. El proceso de volverse sexualmente alfabetizado vincula a las personas con comunidades más grandes y, en última instancia, con la sociedad, apoyando el desarrollo individual y la democracia” (Herdt y Howe, 2007, p. 3).

Sin embargo, es necesario llevar estas discusiones a otros entornos, principalmente a la academia, al contexto familiar, al contexto gubernamental, a la comunidad en un sentido más amplio, a los espacios de diálogo inter e intracultural entre las mayorías y las minorías. Es precisamente en este diálogo franco derivado de sus propias cosmovisiones y de sus prácticas discursivas que se construye la identidad colectiva de una comunidad.

Finalmente, es urgente construir pedagogías que abarquen los postulados posestructuralistas, de modo que la construcción de una identidad sexual, así como cualquier otra identidad, esté sujeta al análisis en términos del impacto que dichas identidades tienen en la enseñanza, en la escuela, en la sociedad y, por supuesto, en el diálogo con y a través de otros grupos culturales. 


\section{Referencias bibliográficas}

Butler, Judith. (1997). Excitable Speech, a Politics of the Performative. Nueva York: Routledge.

Cameron, Deborah y Kulick, Don. (2003). Language and Sexuality. Cambridge: Cambridge University Press.

Castelar, Andrés. (2015). Diversidad, identidad, sexualidad (un palimpsesto). Santiago de Cali: Universidad ICESI.

DeWalt, Kathleen y DeWalt, Billie. (2002). Participant Observation: a Guide for Fieldworkers. Walnut. Creek, CA: AltaMira Press.

Epps, Brad. (2007). Retos y riesgos, pautas y promesas de la teoría Queer. Debate Feminista, 36, 219-272.

Eribon, Didier. (2000). Identidades. Reflexiones sobre la cuestión Gay. (José Miguel Marcén, Trad.) Barcelona: Ediciones Bellaterra.

Foucault, Michel. (1966). Las palabras y las cosas. México D.F.: Siglo XXI.

Foucault, Michel. (1978). The History of Sexuality: An Introduction. Harmondsworth: Penguin Books.

Gagnon, John y Simon, William. (1974). Sexual Conduct: The Social Sources of Human Sexuality. Londres: Hutchinson y Co. Ltd.

Guasch, Oscar. (2000). La crisis de la heterosexualidad. Barcelona: Laertes.

Gray, John. (2016). Language and Non-normative Sexual Identities. En S. Preece (Ed.), The Routledge Handbook of Language and Identity (pp. 225-240). Londres, Reino Unido: Routledge.

Harvey, Keith. (2000). Describing Camp Talk: Language/Pragmatics/Politics. Language and Literature, 9 (3), 240-260. 
Hayes, Joseph. (2006). Gayspeak. En Deborah. Cameron y Don. Kulick (eds). The Language and Sexuality Reader (pp. 68-77). Abingdon: Routledge.

Harsin, Steven. (1991). Pathfinder: Developing a Male Gay Identity. Collection Building, 11(4), 31-36.

Herdt, Gilbert y Howe, Cymene. (2007). 21st Century Sexualities: Contemporary Issues in Health Education and Rights. Nueva York: Routledge.

Kecskes, Istvan. (2018). How Does Intercultural Communication Differ from Intracultural Communication? En Curtis, Andy y Roland Sussex (eds.). Intercultural Communication in Asia: Education, Language and Values (pp. 115-135). Cham: Springer.

Kinsey, Alfred; Pomeroy, Wardell y Martin, Clyde. (1948). Sexual Behavior in the Human Male. Philadelphia: Saunders.

Kroskrity, Paul. (1999). Identity. Journal of Linguistic Anthropology, 9 (1-2), pgs. 111-114.

Kroskrity, Paul. (2000). Regimes of Language: Ideologies, Polities, and Identities. Santa Fe: $\quad$ School of American Research Press.

López, Susana. (2008). El Laberinto Queer, la identidad en tiempos de neoliberalismo. Barcelona: EGALES, S.L.

McIntosh, Mary. (1968). The Homosexual Role. Social Problems, 16 (2). pp. 182-192.

Montes, José. (1983). Motivación y creación léxica en el Español de Colombia. Bogotá: Instituto Caro y Cuervo.

Ramírez, Alexander y Herrera, Rogelio. (2018). El habla rosa: creación léxica en un grupo de hombres homosexuales en Santiago de Cali (Colombia). Lenguaje, 46 (1)41-67.

Urrea, Fernando y La Furcia, Ange. (2014). Pigmentocracia del deseo en el mercado sexual Trans de Cali, Colombia. Sexualidad, Salud y Sociedad, Revista Latinoamericana, 16. 121-152. http://dx.doi.org/10.1590/S1984-64872014000100007. 
Waldner-Haugrud, Lisa y Magruder, Brian. (1996). Homosexual Identity Expression Among Lesbian and Gay Adolescents, an Analysis of Perceived Structural Associations. Youth y Society, 27(3), 313-333.

Wardhaugh, Ronald. y Fuller, Janet. (2014). An Introduction to Sociolinguistics (7 Ed). Oxford: Wiley.

Weeks, Jeffrey (2011). The Languages of Sexuality. Nueva York: Routledge.

Woolard, Kathyn y Schieffelin, Bambi. (1994). Language Ideology. Annual Review of Anthropology, 23, 55-82. 